\title{
Similar Odorants Elicit Different Behavioral and Physiological Responses, Some Supersustained
}

\author{
Shelby A. Montague, ${ }^{1,2}$ Dennis Mathew, ${ }^{2}$ and John R. Carlson ${ }^{2}$ \\ Departments of ${ }^{1}$ Cellular and Molecular Physiology and ${ }^{2}$ Molecular, Cellular, and Developmental Biology, Yale University, New Haven, Connecticut 06520
}

An intriguing question in the field of olfaction is how animals distinguish among structurally similar odorants. We systematically analyzed olfactory responses elicited by a panel of 25 pyrazines. We found that structurally similar pyrazines elicit a wide range of behavioral responses from Drosophila larvae. Each pyrazine was tested against all functional receptors of the larval Odor receptor (Or) repertoire, yielding 525 odorant-receptor combinations. Different pyrazines vary markedly in the responses they elicit from the Or repertoire, with most strong responses deriving from two receptors, Or33b and Or59a. Surprisingly, 2-ethylpyrazine and 2-methylpyrazine, which elicit strikingly similar physiological responses across the receptor repertoire, elicit dramatically different behavioral responses. A small fraction of odorant-receptor combinations elicit remarkably long responses. These responses, which we term "supersustained" responses, are receptor specific and odorant specific, and can last for minutes. Such supersustained responses may prevent olfactory neurons from reporting contemporaneous information about the local odor environment. Odors that elicit such responses could provide a novel means of controlling insect pests and vectors of human disease by impairing the location of human hosts, food sources, and mates.

\section{Introduction}

How are odorants detected and identified by olfactory receptors, neurons, and circuits? This problem has been approached largely through comparison of responses to chemically diverse odorants. Much less attention has been paid to responses elicited by chemically similar odorants. However, the ability to discriminate among structurally similar odorants, including the ability to identify species-specific pheromones, is essential to the survival of many animal species (Larsson et al., 2001, 2002; Nikonov and Leal, 2002; Nikonov et al., 2002).

The Drosophila larva provides an excellent model system for determining how chemical information is encoded and translated into behavioral responses. The larva expresses only 25 Odor receptor (Or) genes (Couto et al., 2005; Fishilevich et al., 2005; Kreher et al., 2005), of which 21 have been found to be functional in a Canton-S strain (Kreher et al., 2005, 2008). There are 21 olfactory receptor neurons (ORNs), which project to 21 glomeruli in the larval antennal lobe, where they synapse with $\sim 21$ projection neurons (Ramaekers et al., 2005). The physiological responses of the Or repertoire to a panel of 26 odorants were recently examined using an in vivo expression system; behavioral responses were also analyzed (Kreher et al., 2008). The odorants were selected largely for their chemical diversity. They included ketones, aromatics, alcohols, esters, aldehydes, a terpene, and an organic acid, and they included odorants of varying carbon chain lengths.

Received Nov. 30, 2010; revised March 25, 2011; accepted April 13, 2011.

Author contributions: S.A.M., D.M., and J.R.C. designed research; S.A.M. performed research; S.A.M. analyzed data; S.A.M., D.M., and J.R.C. wrote the paper.

This work was supported by an NIH National Research Service Award predoctoral grant (to S.A.M.) and by grantsfrom the $\mathrm{NIH}$ (to J.R.C.). We thank Carlotta Martelli for writing the Matlab spike sorting routine and for discussion of data analysis, Thierry Emonet for helpful discussions, and members of the Carlson lab for comments on the manuscript.

Correspondence should be addressed to John R. Carlson at the above address. E-mail: john.carlson@yale.edu.

DOI:10.1523/JNEUROSCI.6254-10.2011

Copyright $\odot 2011$ the authors $\quad 0270-6474 / 11 / 317891-09 \$ 15.00 / 0$
In the present study, we examined olfactory responses elicited by odorants selected for their chemical similarity. Specifically, we analyzed responses elicited by 25 pyrazines, a class of compounds found in extracts of yeast (Ames and Elmore, 1992), which is a major Drosophila food source, in wines (Lacey et al., 1991) and in other sources. Pyrazines also act as pheromones in a variety of insect species (Woolfson and Rothschild, 1990). For example, pyrazines act as attractive pheromones in ladybird beetles (Al Abassi et al., 1998) and as alarm pheromones in ants (Wheeler and Blum, 1973; Vander Meer et al., 2010).

Here we show that, despite their chemical similarity, behavioral responses to the 25 pyrazines vary widely across a continuum, ranging from strong attractive responses to no response at all in a classic behavioral paradigm. We tested each pyrazine against all 21 Ors. Different pyrazines evoked diverse responses from individual receptors, ranging from strong excitation to inhibition. Surprisingly, two pyrazines that elicited dramatically different behavioral responses elicited very similar responses from the Or repertoire, both in terms of response magnitude and response dynamics. A systematic analysis of response dynamics reveals that two receptors, Or33b and Or59a, yield remarkably long-lasting responses to certain pyrazines. In some cases, a $0.5 \mathrm{~s}$ odor stimulus evokes a response that lasts for minutes, illustrating the capacity of the ORNs to encode odorants not only via the magnitudes of their responses but also by their temporal dynamics.

\section{Materials and Methods}

Drosophila stocks. All $\Delta$ halo;UAS-OrX lines and the $\Delta$ halo;Or22a-GAL4 line were described previously (Dobritsa et al., 2003; Hallem et al., 2004; Kreher et al., 2005, 2008). A Canton-S line was used for behavioral experiments. The Orco $^{l}$ mutant (Drosophila Stock Center, Bloomington, IN) was backcrossed to a $w$ Canton-S line for 10 generations before behavioral testing.

Odorants. Pyrazines were from a subsidiary of Sigma, SAFC, and were among a collection of flavors and fragrances. Odorants were obtained at the 
highest available purity and were dissolved in paraffin oil, except for 2-acetylpyrazine, which was dissolved in water. Liquid odorants were diluted to a $10^{-2}$ vol:vol dilution, and solid odorants $[2,3,5,6-$ tetramethylpyrazine, 2-acetyl-3-methylpyrazine, 2-acetyl-3,5(or 6)-dimethylpyrazine, 2,6-dimethylpyrazine, pyrazine, and 2-acetylpyrazine] were used at $10 \mathrm{mg} / \mathrm{ml}$.

Behavioral assays. Behavioral assays were conducted as described previously (Monte et al., 1989; Kreher et al., 2008). Odor was added to a filter disc on one side of a Petri dish and the diluent was added to a filter disc on the opposite side. After $5 \mathrm{~min}$, the number of larvae on each half of the dish was counted to generate the response index (RI). All odorants were tested at a $10^{-2}$ dilution unless otherwise indicated. For the masking experiment (Kreher et al., 2008), a $10^{-2}$ dilution of 2-ethylpyrazine was used as the point source, and a $10^{-2}$ dilution of 2-methylpyrazine was used as the masking odor. As a control, we used the paraffin oil diluent as a masking stimulus.

Electrophysiology. Empty neuron recordings were conducted as previously described (Dobritsa et al., 2003; Kreher et al., 2005), except that a tungsten recording electrode was used in place of a glass recording electrode. Recordings were performed on male and female flies aged 3-10 d. Odors were presented at a rate of $0.2 \mathrm{~L} / \mathrm{min}$ into a constant air stream of $2 \mathrm{~L} / \mathrm{min}$. Data were recorded using Axoscope 9 (Molecular Devices) or LabVIEW software (National Instruments). Spike sorting was performed offline using custom Matlab (MathWorks) scripts.

For recordings of responses to stimuli delivered in the presence of background odor, a $5 \times$ $10^{-4}$ dilution of 2-ethylpyrazine or 2-methylpyrazine was placed in a $125 \mathrm{ml}$ flask as a source of background odor. The flask was then inserted in series with the constant air stream behind a twoway valve. After preparing the fly and identifying the receptor of interest for recording, the valve was opened to expose the fly to the background odor. Exposure to the background lasted for $>5 \mathrm{~min}$ before presentation of other odor stimuli. Dilutions of the same odor as the background odor were then presented in a series, starting with the $10^{-6}$ dilution and ending with a $10^{-1}$ dilution, superimposed upon the constant background.

Modifications were made in some experiments to minimize the possibility of odor adherence to the odor delivery system. Polytetrafluoroethylene (PTFE) tubing (McMaster-Carr Supply Company), which provides a surface with a low frictional coefficient, was inserted into the glass air delivery tube for the indicated supersustained recordings in Figure 6, $C$ and $D$. In other indicated recordings in Figure 6, $C$ and $D$, odor was presented directly to the fly antenna, bypassing this tube. In the third set of recordings in Figure 6, $C$ and $D$, a vacuum was added opposite the air delivery tube. The vacuum consisted of a 2 -inch-diameter cylinder connected to a central vacuum line by Tygon tubing. A $2 \mathrm{~L}$ beaker was added between the cylinder and the central vacuum line to prevent fluctuations in the suction rate of the central vacuum line from affecting the constant removal of air from the recording set-up.

Data processing and statistical analysis. Principal components analysis of both physicochemical space and biological space was performed with built-in Matlab functions. Physicochemical odor space was constructed using 32 optimized chemical descriptors (Haddad et al., 2008) obtained from DRAGON (Talete, srl, DRAGON for Windows, version 5.5, 2007, http://www.talete.mi.it/). Descriptors were normalized across a set of $>500$ odors by dividing the value of each descriptor by its maximum value across all odors minus its minimum value across all odors: normalized descriptor $=$ descriptor $/(\max$ value - min value $)$. Euclidean distance was calculated using Matlab functions.

Analysis of physiological recordings was performed offline using custom Matlab scripts. Recordings were excluded from the analysis if either no spikes could be distinguished after the odor presentation due to pinching (a severe decrease in the amplitude of the spikes) or if A and $\mathrm{B}$ spikes could not be differentiated during any part of the recordings. Overall $\sim 4 \%$ of recordings were excluded for these reasons, and $\sim 19 \%$ of supersustained recordings were excluded due to their greater tendency to pinch. Physiological responses were calculated by subtracting the baseline firing rate and response to the diluent from each response. Peristimulus time histograms (PSTHs) in Figure $6 B-D$ were calculated in Matlab using a $1 \mathrm{~s}$ bin size with a $100 \mathrm{~ms}$ sliding window. PSTHs in Figure $5 C$ were calculated in Matlab using a $50 \mathrm{~ms}$ bin size with a $25 \mathrm{~ms}$ sliding window, as described previously (Bhandawat et al., 2007). Responses 


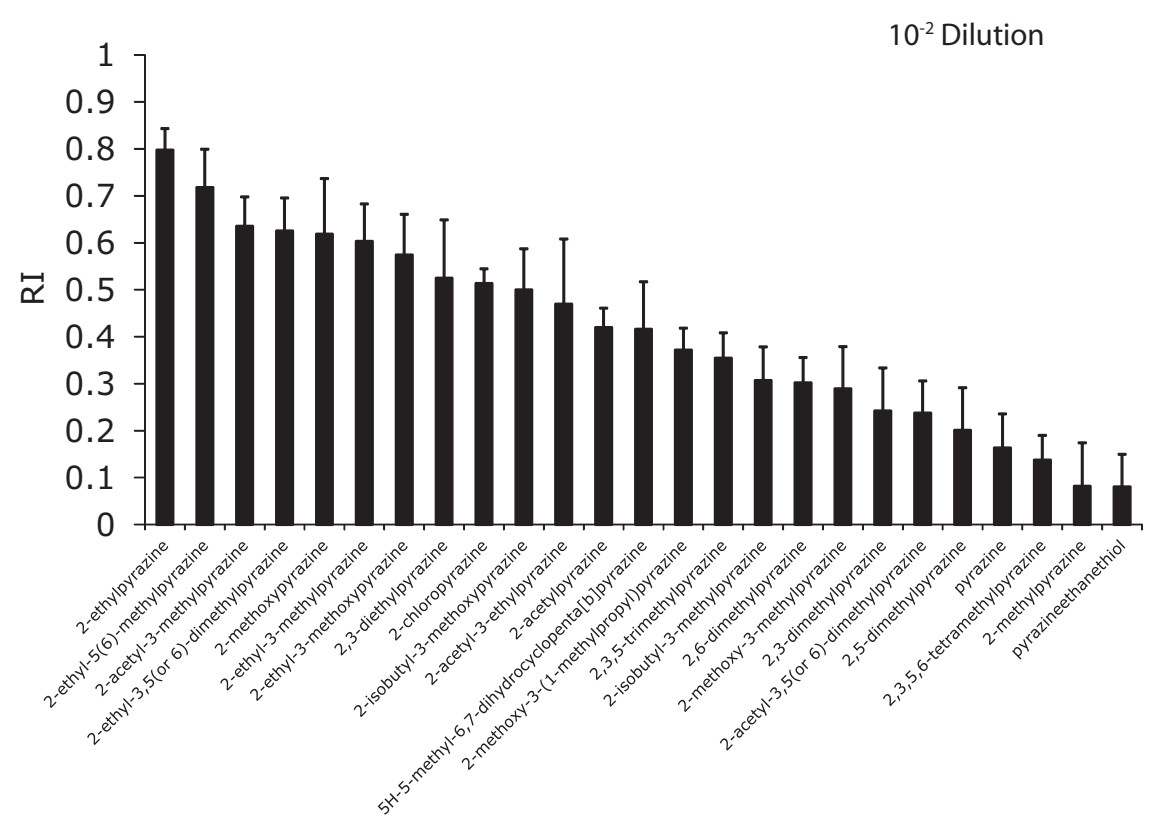

Figure 2. Pyrazines elicit a range of behavioral responses in a two-choice larval behavioral paradigm. Odorants were presented as $10^{-2}$ dilutions. $n=6-8$; error bars are SEM.

were defined as "supersustained" if the firing frequency during the $0.5 \mathrm{~s}$ period that begins $9 \mathrm{~s}$ after the onset of the $0.5 \mathrm{~s}$ odor stimulus period was two SDs above the spontaneous firing frequency. The entire recording interval, including the prestimulus period, was $11 \mathrm{~s}$ in the initial pyrazine screen in which the supersustained responses were first identified.

One-way ANOVA was used to test the statistical significance of the receptor response profiles of 2-ethylpyrazine and 2-methylpyrazine, and two-way ANOVA was used to test the significance of the dose-response curves for 2-ethylpyrazine and 2-methylpyrazine using R software (version 2.11.0, 2010).

\section{Results}

\section{Pyrazines cluster in odor space}

We compared the chemical properties of the 25 pyrazines to those of 109 odorants that had previously been tested against larval odor receptors (Hallem and Carlson, 2006; Kreher et al., 2008). We mapped all of these odorants into a 32-dimensional odor space, in which each dimension represents one of an optimized set of 32 molecular descriptors, including functional group, carbon chain length, and other physicochemical properties (Haddad et al., 2008). Each odorant maps to a particular location in this 32-dimensional space. Odorants that are structurally similar map close together; odorants that are dissimilar map far apart. To visualize the space we projected it into three dimensions via principal component analysis (PCA).

The pyrazines cluster in odor space (Fig. 1), demonstrating their high degree of chemical similarity. Moreover, they map to a region of odor space that was not explored in these previous studies.

\section{Pyrazines elicit a wide range of larval behavioral responses}

We tested each of the 25 pyrazines in a simple two-choice behavioral paradigm in which larvae were allowed to migrate on the surface of an agarose Petri plate toward a source of odorant (Rodrigues and Siddiqi, 1978). An aliquot of odorant was placed on one side of the plate and $\sim 50$ larvae were placed in the center of the plate. After $5 \mathrm{~min}$, an RI was calculated by counting the number of animals, $S$, on the half of the plate containing the odorant, subtracting the number of animals, $C$, on the control half, and dividing by the total number of animals. Thus RI $=(S-C) /(S+C)$. Complete attraction produces an RI of 1 ; indifference produces an RI of 0 .

Despite their chemical similarity, the pyrazines elicited a wide range of behavioral responses (Fig. 2). At one extreme, 2-ethylpyrazine elicited a strong attractive response $(\mathrm{RI}=0.80 \pm 0.04 ; n=6)$. At the other extreme, 2-methylpyrazine and pyrazineethanethiol showed little if any attractive response $(\mathrm{RI}=0.08 \pm 0.10$ and $0.08 \pm 0.07$, respectively; $n=6$ ). We note that the much greater response to 2-ethylpyrazine than 2-methylpyrazine, to which it is chemically similar, is not due to a greater vapor pressure; in fact, 2-ethylpyrazine has a lower vapor pressure (1.67 vs $8.06 \mathrm{mmHg}$ at $\left.25^{\circ}\right)$ and a greater molecular weight (108 vs $94 \mathrm{~g} / \mathrm{mol}$ ). Moreover, a systematic analysis did not reveal a correlation between RI and the vapor pressure $\left(R^{2}=0.12\right)$ or boiling point $\left(R^{2}=0.01\right)$ of the pyrazines in our panel.

\section{Coding of pyrazines by the larval odor receptor repertoire}

To understand how the structurally similar pyrazines are encoded across the larval receptor repertoire, we systematically tested each of the 25 pyrazines against the 21 Ors that have been previously shown to be functional in our Canton-S strain (Kreher et al., 2008), giving us a total of 525 odorant-receptor combinations. We tested them in the empty-neuron system, which is based on a mutant ORN of the fly antenna that lacks a functional endogenous receptor (Dobritsa et al., 2003; Hallem et al., 2004). Individual larval Or genes are expressed in this ORN and the olfactory responses that each imparts are determined by single-unit electrophysiological recordings. Previous work has shown that the empty neuron is a faithful expression system for Or genes from the adult fly (Hallem et al., 2004) and mosquito (Carey et al., 2010) and that analysis of larval Or genes in the empty neuron agrees with the phenotypes of certain larval Or mutations (Kreher et al., 2008).

Different pyrazines varied markedly in the responses they elicited from the receptor repertoire: 2-ethylpyrazine (Fig. $3 A$, top row, and Table 1$)$, elicited strong or moderate responses $(++$ or + , defined as $\geq 100$ or $\geq 50$ spikes/s, respectively) from five receptors at the test concentration; pyrazineethanethiol (Fig. $3 A$, bottom row) elicited none. Individual pyrazines elicited excitatory responses from some receptors and inhibitory responses from others.

Strikingly, two receptors, Or33b and Or59a, accounted for most of the strong responses. The identification of Or33b as a receptor that responds strongly to pyrazines was particularly notable in that Or33b had yielded no excitatory responses of even moderate strength to any of $>100$ odorants tested in previous studies (Hallem and Carlson, 2006; Kreher et al., 2008). Its strong response to 10 of 25 pyrazines tested (Fig. $3 A,++$ ), but to none of the other odorants, suggests that it evolved to detect pyrazines.

To quantitate the diversity in responses elicited by the various pyrazines, we mapped them in a 21 -dimensional biological odor space, in which each dimension represented the response magnitude elicited by each of the 21 receptors. We then considered the Euclidean distances between individual pyrazines in this space. 
A

2-ethylpyrazine
2-ethyl-5(6)-methylpyrazine
2-acetyl-3-methylpyrazine
2-ethyl-3,5(or 6)-dimethylpyrazine
2-methoxypyrazine
2-ethyl-3-methylpyrazine
2-ethyl-3-methoxypyrazine
2,3-diethylpyrazine
2-chloropyrazine
2-sobutyl-3-methoxypyrazine
2-acetyl-3-ethylpyrazine
2-acetylpyrazine
5H-5-methyl-6,7-dihydrocyclopenta[b]p.
2-methoxy-3-(1-methylpropyl)pyrazine
2,3,5-trimethylpyrazine
2-sobutyl-3-methylpyrazine
2,6-dimethylpyrazine
2-methoxy-3-methylpyrazine
2,3-dimethylpyrazine
2-acetyl-3,5(or 6)-dimethylpyrazine
2,5-dimethylpyrazine
pyrazine
2,3,5,6-tetramethylpyrazine
2-methylpyrazine
pyrazineethanethiol

2a 7a 13a 22c 24a 30a 33b 35a 42a 42b 45a 45b 47a 49a 59a 67b 74a 82a 85c 94a 94b

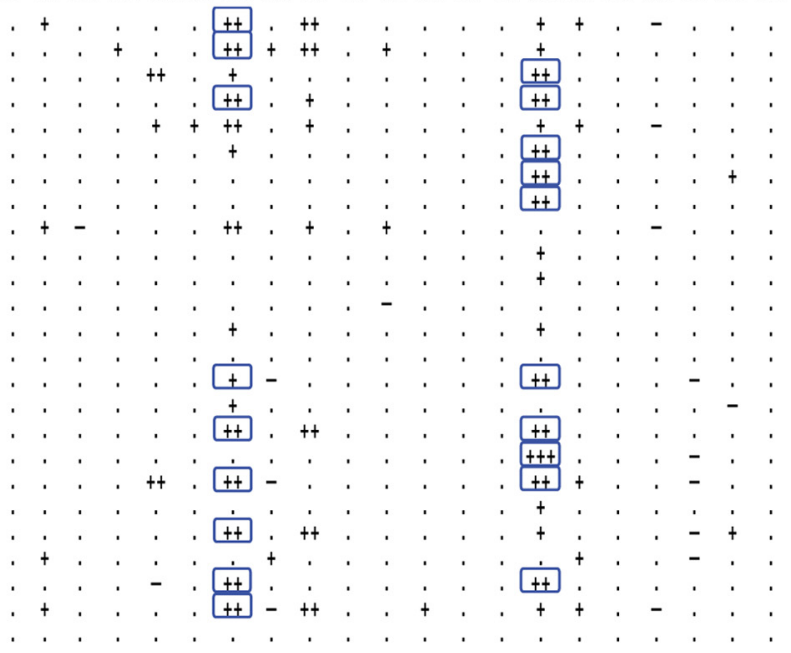

\section{B}

2-ethylpyrazine

2-ethyl-5(6)-methylpyrazine 2-acetyl-3-methylpyrazine

2-ethyl-3,5(or 6)-dimethylpyrazine 2-methoxypyrazine

2-ethyl-3-methylpyrazine 2-ethyl-3-methoxypyrazine 2,3-diethylpyrazine 2-chloropyrazine 2-isobutyl-3-methoxypyrazine 2-acetyl-3-ethylpyrazine 2-acetylpyrazine

5H-5-methyl-6,7-dihydrocyclopenta[b]p. 2-methoxy-3-(1-methy|propy))pyrazine 2,3,5-trimethylpyrazine 2-isobuty|-3-methylpyrazine 2,6-dimethylpyrazine 2-methoxy-3-methylpyrazine 2,3-dimethylpyrazine 2-acetyl-3,5(or 6)-dimethylpyrazine 2,5-dimethylpyrazine pyrazine

2,3,5,6-tetramethylpyrazine 2-methylpyrazine pyrazineethanethiol
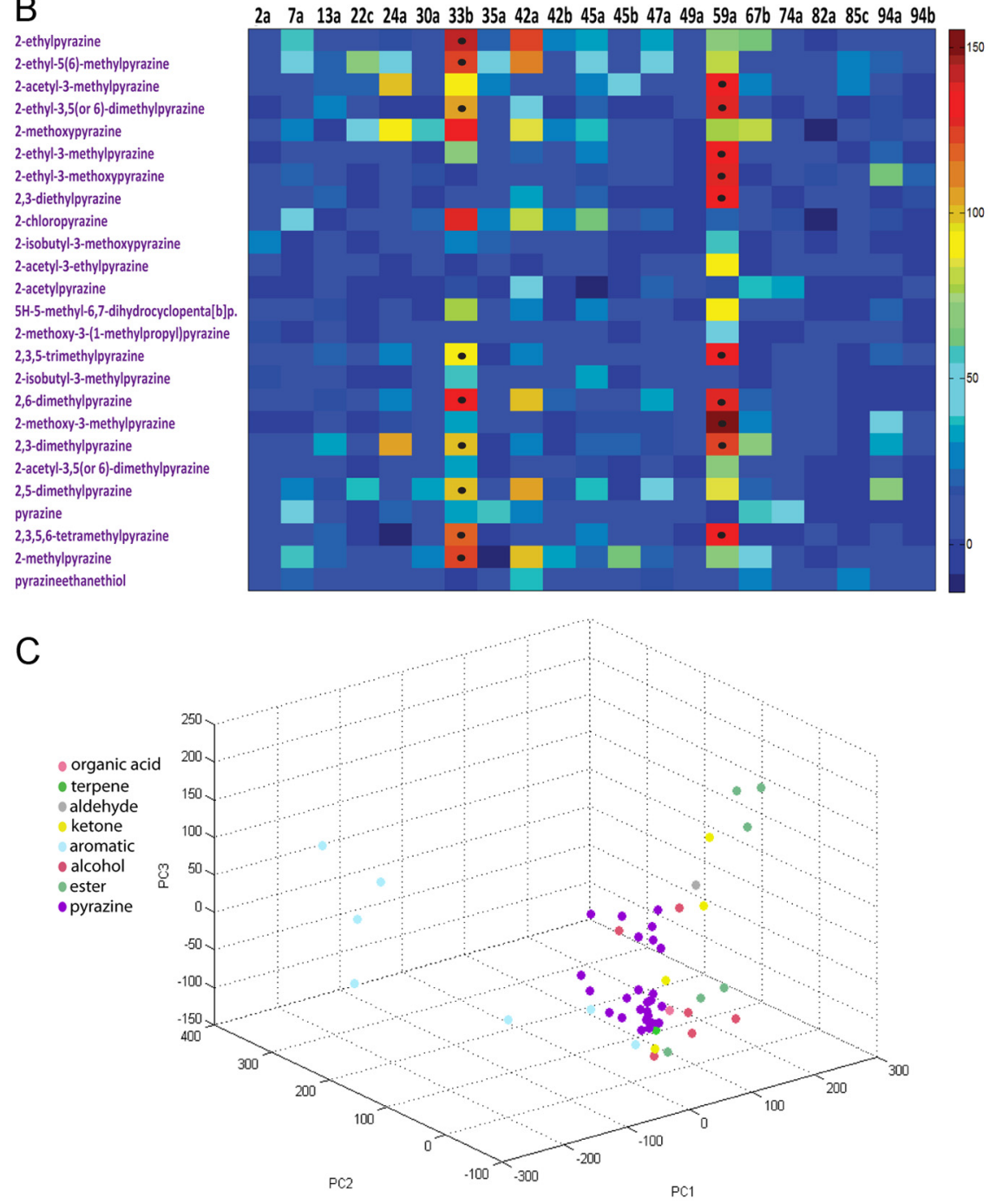

Figure 3. Responses of larval odor receptors to pyrazines. $\boldsymbol{A}$, Responses to pyrazines. - , Inhibition;,$n<50$ spikes $/ \mathrm{s} ;+, 50 \leq$ $n<100$ spikes/s;,$++ 100 \leq n<150$ spikes/s;,$+++ n \geq 150$ spikes/s. Response frequencies were calculated by counting the number of spikes during the $0.5 \mathrm{~s}$ odor presentation period and multiplying by two to determine the number of impulses per second, then subtracting the background firing rate and the response to the diluent alone. We have defined an inhibitory response as one that is at least one SD below the receptor's baseline firing rate, after the mean response to the diluent control and the background firing rate were subtracted from the response. Blue boxes indicate that a response was supersustained, defined as a response rate at least 2 SDs above the baseline firing rate $9 \mathrm{~s}$ after the 0.5 s odor presentation period. 0 dors were presented as $10^{-2}$
The distances ranged from 37 to 223 spikes/s, with a mean distance of $134 \pm 51$ spikes/s (SD; $n=300$ pairs). We then compared these distances to corresponding distances obtained with a panel of 26 diverse odorants (Kreher et al., 2008). In that study, the distances ranged from 82 to 603 spikes/s, with a mean of $347 \pm 131$ spikes/s (SD; $n=325$ pairs). The simplest interpretation of the greater mean distance between the diverse odors $(p<$ 0.0001 ) is that it reflects a greater diversity in their representation among the receptor repertoire. However, in principle, the greater mean distance could arise solely from experimental differences between the two studies that produced greater response magnitudes in Kreher et al. (2008). We therefore expressed each response magnitude in terms of the proportion of the maximal response obtained in each study. The distances were again greater among the diverse odorants than among the pyrazines when expressed in this way $(p<0.0001)$. A three-dimensional representation of this 21-dimensional biological odor space was produced by PCA (Fig. $3 C)$. We note with interest that although pyrazines cluster tightly in a region removed from other odorants in physiochemical odor space (Fig. $1 A$ ), they do not in this biological odor space.

Many of the strong responses of Or33b and Or59a showed remarkable temporal dynamics, showing long-lasting firing that persisted well past the end of the odor stimulus period. These responses are indicated by boxes in Figure $3 A$ and are considered in detail below.

\section{Two odorants elicited similar Or responses but dramatically different behavior}

We were surprised to find that 2ethylpyrazine and 2-methylpyrazine,

\section{$\leftarrow$}

dilutions. All odor-receptor pairs were initially tested three times; odor-receptor pairs that elicited an average response of $>20$ or $<0$ spikes/s were then tested an additional three times, for a total of six tests. Numerical values for each receptor-odorant combination are given in Table 1. 5H-5-methyl-6,7-dihydrocyclopenta[b]p., 5H-5Methyl-6,7-dihydrocyclopenta[b]pyrazine. B, Heat map of larval receptor responses to pyrazines in spikes per second. The data are the same as in $\boldsymbol{A}$, with the diluent response and background firing rate again subtracted from each response. Supersustained responses, defined as in $\boldsymbol{A}$, are marked with dots. C, Three-dimensional representation, generated by PCA, of a 21-dimensional biological odor space. Vectors quantifying the responses of the 21 receptors to each tested odor were projected onto a threedimensional space, which captures $65 \%$ of the variation in the original 21-dimensional dataset. 
Table 1. Responses of receptors to pyrazines at a $10^{-2}$ dilution

\begin{tabular}{|c|c|c|c|c|c|c|c|c|c|c|c|c|c|c|c|c|c|c|c|c|c|}
\hline & $0 \mathrm{r} 2 \mathrm{a}$ & Or7a & Or13a & $0 \mathrm{r} 22 \mathrm{c}$ & Or24a & Or30a & $0 \mathrm{r} 33 \mathrm{~b}$ & $0 \mathrm{r35a}$ & Or42a & Or42b & Or45a & Or45b & Or47a & Or49a & Or59a & Or67b & Or74a & Or82a & Or85c & Or94a & Or94b \\
\hline ine & $9 \pm 1$ & $56 \pm 13$ & $9 \pm 5$ & \pm 9 & $16 \pm 9$ & $13 \pm 3$ & $144 \pm 9$ & $18 \pm 5$ & $124 \pm 10$ & $24 \pm 15$ & $35 \pm 5$ & $12 \pm 7$ & $35 \pm 9$ & $10 \pm 2$ & $66 \pm 12$ & $60 \pm 14$ & $7 \pm 5$ & $-6 \pm 8$ & $9 \pm 11$ & $2 \pm 8$ & $8 \pm 8$ \\
\hline methylpyrazine & $14 \pm 4$ & $47 \pm 12$ & $22 \pm 4$ & $68 \pm$ & \pm 46 & $7 \pm 5$ & $3 \pm 23$ & \pm 11 & $114 \pm 13$ & $1 \pm 9$ & $1 \pm 16$ & $11 \pm 11$ & $41 \pm 12$ & $5 \pm 5$ & $79 \pm 23$ & $15 \pm 4$ & \pm 1 & $9 \pm 5$ & $26 \pm 3$ & & $9 \pm 9$ \\
\hline 2-Acetyl-3-methylpyrazine & $11 \pm 4$ & $8 \pm 2$ & $18 \pm 1$ & $18 \pm 12$ & $101 \pm 28$ & $7 \pm 3$ & $90 \pm 20$ & $25 \pm 9$ & $9 \pm 5$ & $3 \pm 9$ & $27 \pm 12$ & $41 \pm 13$ & $12 \pm 5$ & $4 \pm 2$ & $132 \pm$ & $24 \pm 7$ & $8 \pm 5$ & $3 \pm 8$ & $25 \pm 21$ & $12 \pm 5$ & $0 \pm 0$ \\
\hline 2-Ethyl-3,5(or 6)-dimethylpyrazine & $1 \pm 2$ & $4 \pm 11$ & $27 \pm 4$ & $3 \pm 7$ & $0 \pm 4$ & $1 \pm 1$ & $105 \pm 23$ & $2 \pm 3$ & $51 \pm 10$ & $0 \pm 1$ & $20 \pm 5$ & $0 \pm 0$ & $16 \pm 15$ & $2 \pm 2$ & $139 \pm 14$ & $2 \pm 2$ & $0 \pm 2$ & $13 \pm 5$ & $0 \pm 12$ & $17 \pm 10$ & $0 \pm 0$ \\
\hline 2-Methoxypyrazine & $10 \pm 2$ & $26 \pm 10$ & $-5 \pm 2$ & $48 \pm 13$ & $94 \pm 28$ & $55 \pm 8$ & $130 \pm 14$ & $11 \pm 11$ & $85 \pm 20$ & $28 \pm 19$ & $37 \pm 10$ & $11 \pm 6$ & $11 \pm 11$ & $1 \pm 1$ & $78 \pm 15$ & $83 \pm 23$ & $7 \pm 5$ & $-13 \pm 5$ & $8 \pm 28$ & $15 \pm 14$ & $11 \pm 11$ \\
\hline ylpyrazine & $1 \pm 5$ & $11 \pm 8$ & $14 \pm 2$ & $2 \pm 3$ & $5 \pm 3$ & $0 \pm 4$ & $69 \pm 14$ & $10 \pm 4$ & $22 \pm 4$ & $3 \pm 8$ & $25 \pm 5$ & $2 \pm 3$ & $9 \pm 4$ & $1 \pm 1$ & $133 \pm 21$ & $7 \pm 2$ & $5 \pm 1$ & $2 \pm 5$ & $1 \pm 3$ & $22 \pm 11$ & $0 \pm 0$ \\
\hline 2-Ethyl-3-methoxypyrazine & $9 \pm 2$ & $19 \pm 12$ & $4 \pm 7$ & $-1 \pm 7$ & $3 \pm 6$ & $15 \pm 7$ & $-5 \pm 4$ & $4 \pm 4$ & $10 \pm 2$ & $10 \pm 8$ & $17 \pm 4$ & $5 \pm 5$ & $12 \pm 5$ & $17 \pm 5$ & $128 \pm 19$ & $1 \pm 2$ & $5 \pm 4$ & $17 \pm 7$ & $12 \pm 11$ & $64 \pm 6$ & $19 \pm 16$ \\
\hline 2,3-Diethylpyrazine & $7 \pm 7$ & $10 \pm 11$ & $18 \pm 5$ & $1 \pm 5$ & $9 \pm 3$ & $1 \pm 1$ & $5 \pm 7$ & $4 \pm 4$ & $32 \pm 8$ & $4 \pm 14$ & $21 \pm 6$ & $0 \pm 0$ & $4 \pm 21$ & $1 \pm 1$ & $134 \pm 20$ & $6 \pm 5$ & $6 \pm 10$ & $9 \pm 4$ & $5 \pm 4$ & $-4 \pm 2$ & $15 \pm 15$ \\
\hline 2-Chloropy & $14 \pm 10$ & $50 \pm 4$ & $-7 \pm 2$ & $8 \pm 1$ & $11 \pm 3$ & $18 \pm 17$ & $127 \pm 21$ & $25 \pm 16$ & $80 \pm 13$ & $28 \pm 7$ & $62 \pm 20$ & $11 \pm 7$ & $19 \pm 20$ & $1 \pm 1$ & & $4 \pm 3$ & $17 \pm 8$ & $-14 \pm 6$ & $2 \pm 21$ & $16 \pm 12$ & $0 \pm 0$ \\
\hline ethoxypyrazine & $28 \pm 5$ & $-5 \pm 7$ & $13 \pm 11$ & $0 \pm 1$ & $6 \pm 4$ & $3 \pm 2$ & $29 \pm 17$ & $21 \pm 1$ & $12 \pm 5$ & $4 \pm 6$ & $2 \pm 7$ & $0 \pm 0$ & $3 \pm 11$ & $1 \pm 1$ & $58 \pm 13$ & $1 \pm 2$ & $5 \pm 7$ & $0 \pm 5$ & $3 \pm 3$ & $0 \pm 6$ & $1 \pm 1$ \\
\hline 2-Acety & $2 \pm 6$ & $1 \pm 5$ & $11 \pm 3$ & $3 \pm 5$ & $1 \pm 3$ & $7 \pm 6$ & $-3 \pm 4$ & \pm 13 & $1 \pm 3$ & $5 \pm 11$ & $2 \pm 4$ & $0 \pm 0$ & $6 \pm 4$ & $2 \pm 1$ & $87 \pm 22$ & $1 \pm 2$ & $6 \pm 7$ & $7 \pm 6$ & $2 \pm 3$ & $13 \pm 8$ & $0 \pm 0$ \\
\hline 2-Acetylp & \pm 5 & $0 \pm 20$ & $5 \pm 7$ & $0 \pm 10$ & $0 \pm 14$ & $0 \pm 3$ & & $0 \pm 13$ & $46 \pm 14$ & $0 \pm 0$ & $-13 \pm 12$ & $0 \pm 3$ & $0 \pm 9$ & $0 \pm 1$ & & $0 \pm 3$ & $0 \pm 13$ & $0 \pm 13$ & $0 \pm 5$ & & $0 \pm 2$ \\
\hline cyclopenta & a $2 \pm 4$ & $8 \pm 6$ & $17 \pm 4$ & $-1 \pm 8$ & $5 \pm 6$ & $1 \pm 1$ & $77 \pm 12$ & $8 \pm 2$ & $23 \pm 5$ & $-6 \pm 8$ & & $9 \pm 5$ & $13 \pm 3$ & $0 \pm 0$ & $88 \pm 20$ & $5 \pm 3$ & & & $0 \pm$ & $18 \pm 13$ & $-2 \pm 3$ \\
\hline 2-Methoxy-3-(1-methylpropyl & $15 \pm 7$ & $-1 \pm 8$ & $3 \pm 3$ & $1 \pm 5$ & $2 \pm 5$ & $3 \pm 1$ & $3 \pm 4$ & $4 \pm 2$ & $7 \pm 3$ & $3 \pm 8$ & $13 \pm 4$ & $1 \pm 1$ & $3 \pm 10$ & $3 \pm 3$ & $48 \pm 21$ & $0 \pm 2$ & $-1 \pm 5$ & $7 \pm 10$ & $6 \pm 4$ & $3 \pm 6$ & $0 \pm 0$ \\
\hline $2,3,5$ & $9 \pm 2$ & $12 \pm 3$ & $23 \pm 5$ & $7 \pm 5$ & $30 \pm 9$ & $3 \pm 1$ & $89 \pm 14$ & $-6 \pm 15$ & $28 \pm 7$ & $4 \pm 5$ & \pm 2 & $6 \pm 6$ & $6 \pm 5$ & $3 \pm 2$ & $135 \pm 13$ & $8 \pm 8$ & $5 \pm 5$ & $4 \pm 5$ & $-8 \pm 3$ & $23 \pm 9$ & $9 \pm 9$ \\
\hline pyrazine & $15 \pm 4$ & $12 \pm 9$ & $15 \pm 4$ & $0 \pm 4$ & $1 \pm 1$ & $2 \pm 0$ & $60 \pm 26$ & $5 \pm 4$ & $3 \pm 3$ & $7 \pm 12$ & $34 \pm 7$ & $0 \pm 0$ & $8 \pm 2$ & $2 \pm 2$ & $13 \pm 5$ & $10 \pm 6$ & $7 \pm 7$ & $7 \pm 5$ & $1 \pm 4$ & $-9 \pm 2$ & $0 \pm 0$ \\
\hline $2,6-\mathrm{L}$ & $11 \pm 5$ & $12 \pm 12$ & $16 \pm 54$ & $4 \pm$ & $27 \pm$ & $11 \pm 4$ & $130 \pm 13$ & $5 \pm 4$ & $102 \pm 19$ & $18 \pm 8$ & \pm 11 & $9 \pm 6$ & $32 \pm 15$ & $7 \pm 3$ & & $20 \pm$ & $11 \pm 6$ & $5 \pm 3$ & $-5 \pm 7$ & $-7 \pm 5$ & $0 \pm 0$ \\
\hline Ipyrazine & $0 \pm 9$ & $1 \pm 2$ & $2 \pm 6$ & $-4 \pm 5$ & $15 \pm 8$ & $8 \pm 3$ & $31 \pm 11$ & $8 \pm 6$ & $8 \pm 4$ & $-1 \pm 12$ & $15 \pm 6$ & $16 \pm 15$ & $16 \pm 6$ & $6 \pm 6$ & $155 \pm 7$ & $29 \pm 9$ & $5 \pm 4$ & $11 \pm 9$ & $-8 \pm 5$ & $44 \pm 11$ & $5 \pm 5$ \\
\hline 2,3-Din & $2 \pm 1$ & $6 \pm 14$ & $33 \pm 2$ & $5 \pm 12$ & $103 \pm 20$ & $10 \pm 5$ & $102 \pm 20$ & $-8 \pm 6$ & $24 \pm 8$ & $1 \pm 3$ & $19 \pm 3$ & $19 \pm 11$ & $17 \pm 9$ & $4 \pm 4$ & $126 \pm 16$ & $66 \pm 12$ & $4 \pm 6$ & $5 \pm 1$ & $-8 \pm 4$ & $33 \pm 7$ & $4 \pm 5$ \\
\hline 2-Acetyl-3,5(or 6)-dimethylpyrazine & e $9 \pm 6$ & $2 \pm 16$ & $3 \pm 3$ & $0 \pm 8$ & $8 \pm 4$ & $3 \pm 1$ & $35 \pm 16$ & $-1 \pm 20$ & $10 \pm 2$ & $6 \pm 5$ & $16 \pm 5$ & $1 \pm 1$ & $6 \pm 0$ & $0 \pm 2$ & $68 \pm 12$ & $3 \pm 5$ & $6 \pm 5$ & $1 \pm 3$ & $1 \pm 4$ & $2 \pm 5$ & $-1 \pm 4$ \\
\hline 2,5-Dimeth & $3 \pm 3$ & $31 \pm 9$ & $16 \pm 7$ & $38 \pm 8$ & \pm 2 & $31 \pm 3$ & $102 \pm 33$ & & $103 \pm 11$ & $16 \pm 12$ & & $1 \pm 1$ & $43 \pm 11$ & $14 \pm 2$ & & & & & & $69 \pm 13$ & $0 \pm 0$ \\
\hline & $5 \pm 8$ & $52 \pm 15$ & $11 \pm 2$ & $-2 \pm 8$ & $21 \pm 23$ & $14 \pm 3$ & $36 \pm 24$ & $58 \pm 2$ & $6 \pm 12$ & $5 \pm 10$ & & $5 \pm 4$ & $2 \pm 4$ & $7 \pm 5$ & $-1 \pm 3$ & $57 \pm 8$ & $43 \pm 2$ & $-3 \pm 1$ & $-7 \pm 2$ & $-1 \pm 4$ & $0 \pm 0$ \\
\hline 2,3,5,6-Tetramethylpyrazine & $3 \pm 6$ & $4 \pm 5$ & $23 \pm 3$ & $1 \pm 6$ & $-12 \pm 4$ & $2 \pm 1$ & $119 \pm 21$ & $-4 \pm 26$ & $12 \pm 5$ & $8 \pm 20$ & $25 \pm 12$ & $4 \pm 5$ & $2 \pm 6$ & $1 \pm 1$ & $137 \pm 13$ & $6 \pm 4$ & $4 \pm 11$ & $10 \pm 5$ & $1 \pm 6$ & $9 \pm 12$ & $0 \pm 0$ \\
\hline 2-Methylpyrazine & $2 \pm 6$ & $57 \pm 10$ & $18 \pm 8$ & $5 \pm 10$ & $11 \pm 4$ & $25 \pm 1$ & $124 \pm 11$ & $-11 \pm 32$ & $102 \pm 14$ & $33 \pm 20$ & $21 \pm 7$ & $65 \pm 13$ & $19 \pm 16$ & $55 \pm 3$ & $73 \pm 24$ & $51 \pm 12$ & $11 \pm 6$ & $-6 \pm 5$ & $2 \pm 19$ & $10 \pm 17$ & $6 \pm 6$ \\
\hline Pyrazineethanethiol & $4 \pm 3$ & $21 \pm 20$ & $6 \pm 3$ & $5 \pm 7$ & $7 \pm 10$ & $3 \pm 1$ & $-1 \pm 3$ & $7 \pm 2$ & $37 \pm 11$ & $2 \pm 7$ & $10 \pm 12$ & $7 \pm 17$ & $15 \pm 6$ & $4 \pm 4$ & $3 \pm 3$ & $29 \pm 13$ & $3 \pm 6$ & $2 \pm 4$ & $23 \pm 9$ & $4 \pm 4$ & $11 \pm 11$ \\
\hline & $8 \pm 7$ & $13 \pm 9$ & $17 \pm 6$ & & & $5 \pm 5$ & & & & & & & $5 \pm 5$ & $1 \pm 2$ & & $3 \pm 3$ & $4 \pm 5$ & & $5 \pm 5$ & $15 \pm 7$ & $2 \pm 4$ \\
\hline & $4 \pm 6$ & $21 \pm 7$ & $13 \pm 4$ & & $8 \pm 8$ & $7 \pm 5$ & $16 \pm 10$ & $6 \pm 9$ & $12 \pm 10$ & $2 \pm 3$ & $13 \pm 7$ & $2 \pm 2$ & $3 \pm 5$ & $5 \pm 6$ & $12 \pm 6$ & $3 \pm 2$ & $9 \pm 6$ & $24 \pm 21$ & $5 \pm 2$ & $13 \pm 8$ & $1 \pm 1$ \\
\hline Water & $7 \pm 6$ & $42 \pm 27$ & $15 \pm 7$ & $72 \pm 22$ & $29 \pm 31$ & $3 \pm 4$ & $9 \pm 9$ & $41 \pm 24$ & $20 \pm 9$ & $0 \pm 0$ & $77 \pm 21$ & $26 \pm 60$ & $18 \pm 17$ & $7 \pm 3$ & $8 \pm 6$ & $10 \pm 10$ & $32 \pm 40$ & $21 \pm 21$ & $47 \pm 12$ & $25 \pm 13$ & $3 \pm 5$ \\
\hline
\end{tabular}

Values are mean spike rates \pm SEM except background, which is mean spike rate \pm SD. Odorant-receptor combinations were initially tested three times; combinations that yielded a mean response $>20$ or $<0$ spikes/s were then tested an additional three times; thus $n=3-6$. For background values, $n>80$. Background firing rates and the responses to the diluent alone were subtracted from the responses to pyrazines. Background firing rates were subtracted from the values shown for the diluents. 5H-5-Methyl-6, 7-dihydrocyclopenta, 5H-5-Methyl-6, 7-dihydrocyclopenta[b]pyrazine; 2-Methoxy-3-(1-methylpropyl), 2-methoxy-3-(1-methylpropyl)pyrazine.

A

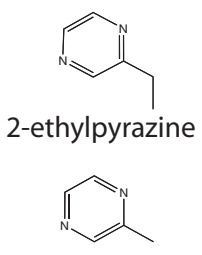

2-methylpyrazine
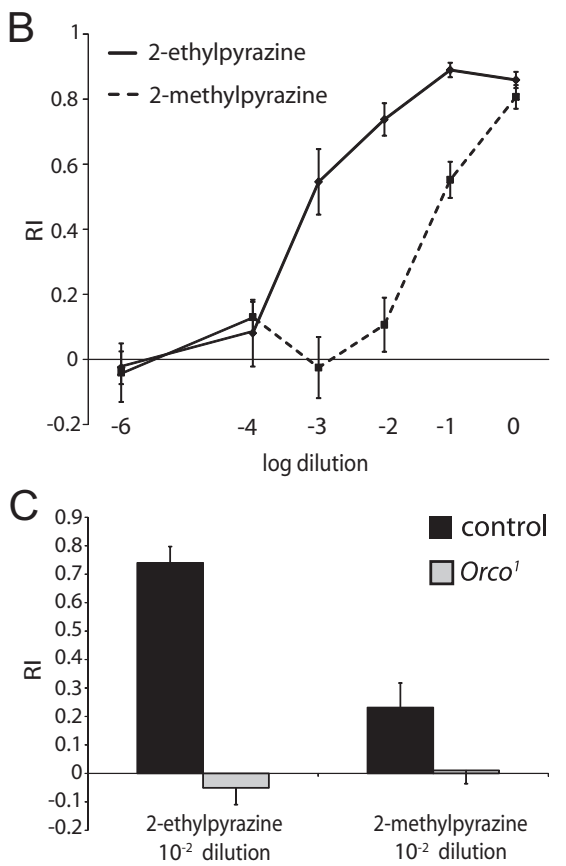

Figure 4. Behavioral responses to 2-ethylpyrazine and 2-methylpyrazine. $A$, Structures of 2-ethylpyrazine and 2-methylpyrazine. B, Behavioral responses to 2-ethylpyrazine (solid line) and 2-methylpyrazine (dashed line) in a two-choice behavioral assay. $n=6$; error bars are SEM. C, Behavioral responses of an olfactory mutant to 2-ethylpyrazine and 2-methylpyrazine at $10^{-2}$ dilutions. Control ( $w$ Canton S) responses are shown in black. The Orco ${ }^{7}$ mutant line (gray) was backcrossed into $w$ Canton $S$ for 10 generations to reduce potential behavioral variation due to differences in genetic background. which elicited dramatically different behavioral responses, elicited remarkably similar physiological responses from the receptor repertoire. We sought to confirm and extend this observation in nine ways.

We tested the behavioral response across a wide range of concentrations of both 2-ethylpyrazine and 2-methylpyrazine. We confirmed that 2-ethylpyrazine elicits a much greater response than 2-methylpyrazine at a $10^{-2}$ dilution and found that it elicited a greater response across a broad range of concentrations (Fig. 4B). Only at the highest concentration tested, at which the response to 2-ethylpyrazine appeared saturated, did the two odorants elicit equally strong attraction. In addition to responding more strongly to 2-ethylpyrazine at most doses, the larva also appears more sensitive to 2-ethylpyrazine; the threshold for response to 2-ethylpyrazine lies between a $10^{-4}$ and a $10^{-3}$ dilution, whereas it is higher for 2-methylpyrazine.

We sought to confirm that the difference in behavioral response to the two pyrazines represents a difference in olfactory response, as opposed to taste response. We repeated the behavioral tests, but with the odorants placed on the lid of the Petri dish so as to prevent the larvae from making contact with them. A striking difference in RI persisted ( $p<0.001$; data not shown), indicating that the behavioral difference relies on a difference in olfactory perception.

We considered the possibility that the difference in olfactory response might arise from a difference in the response of an olfactory receptor that does not belong to the Or family, such as an Ionotropic receptor (Benton et al., 2009; Liu et al., 2010; Abuin et al., 2011). We tested the responses of a mutant defective in Orco (formerly known as Or83b), which is believed to be an essential coreceptor of all Ors (Larsson et al., 2004; Sato et al., 2008; Wicher et al., 2008). The Orco mutant did not respond to either 2-methylpyrazine or 2-ethylpyrazine, supporting the notion that the behavioral response to both odors is due to Ors (Fig. 4C).

Having confirmed that the difference in behavioral response to 2-ethylpyrazine and 2-methylpyrazine arises from a difference in the response of the Or repertoire, we performed an additional, independent analysis of the responses of the Ors to the two pyrazines. The two pyrazines were compared directly in pairwise fashion, such that each fly in the analysis was tested with 
both pyrazines. The responses of the receptor repertoire to the two pyrazines were again very similar. In fact, they were indistinguishable by one-way ANOVA ( $p>$ 0.05) (Fig. 5A).

Next, we examined in more detail the responses of the six receptors that gave at least a moderate response to the odorants at the initial test concentration. We compared the responses of these receptors across a broad range of concentrations (Fig. 5B). The responses were strikingly similar. Although there are some differences in the mean responses at specific dilutions, there was no significant difference between the two doseresponse curves for any receptor (two-way ANOVA, $p>0.05$ ).

We then tested the possibility that the receptors differ in the temporal dynamics of their responses to the two odorants. Previously, we have compared the magnitudes of responses to the two odorants over a $0.5 \mathrm{~s}$ interval; here we considered their dynamics over a period of $3 \mathrm{~s}$. We did not observe dramatic differences between the responses to the two odorants for any receptor (Fig. 5C).

We considered the possibility that the animal might differ in its ability to migrate up gradients of the two odorants. As one initial means of testing this possibility, we examined the Or42a receptor, which responds strongly to both odorants. We exposed Or42a to a prolonged odor stimulus, during the course of which we delivered $0.5 \mathrm{~s}$ pulses of the same odor. Stimuli of concentrations greater than the background elicited responses in the case of each odor, and the magnitudes of these responses were comparable for 2-ethylpyrazine and 2-methylpyrazine across a range of concentrations (data not shown). We also performed a behavioral experiment based on an odormasking paradigm (Kreher et al., 2008) designed to investigate whether the perceptual qualities of the two odors are similar. We found that a background of 2-methylpyrazine reduced the RI to a point source of 2-ethylpyrazine to $\sim 50 \%$ that of the control value, thus suggesting perceptual similarity between the two odors $(p<0.001$; data not shown).

Finally, we also examined two other receptors, Orla and Or33a, which we had earlier found to exhibit polymorphism, i.e., alleles amplified from our Canton S strain were nonfunctional, but alleles found in another strain, Oregon $\mathrm{R}$, varied by a single nucleotide from their Canton S counterparts and were functional. We tested the Oregon $\mathrm{R}$ alleles of both receptors for response to 2-ethylpyrazine and 2-methylpyrazine. Both receptors responded weakly to both odorants at our standard test concentration; each receptor responded to the two odorants with equal magnitudes ( $p>0.05$; data not shown).

B

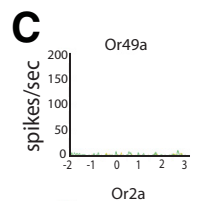

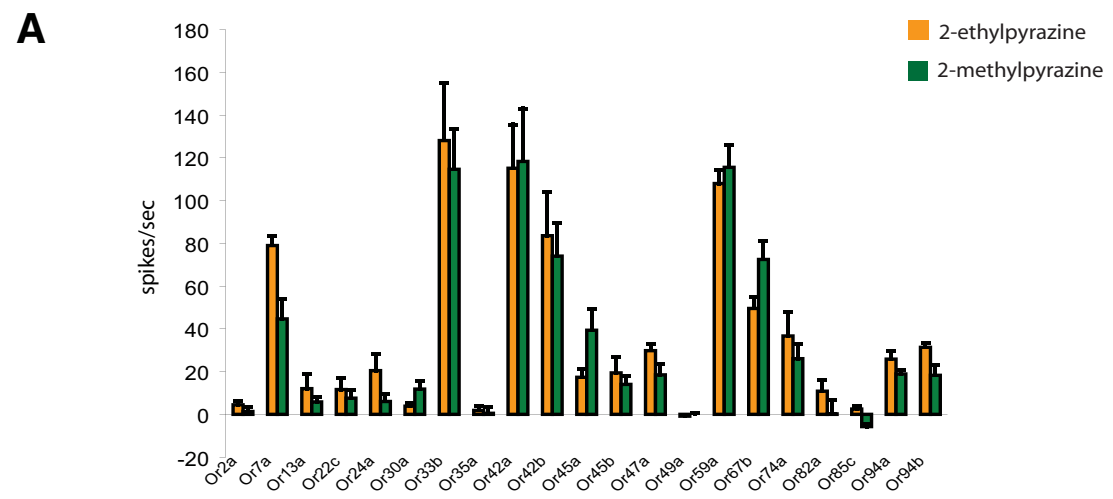
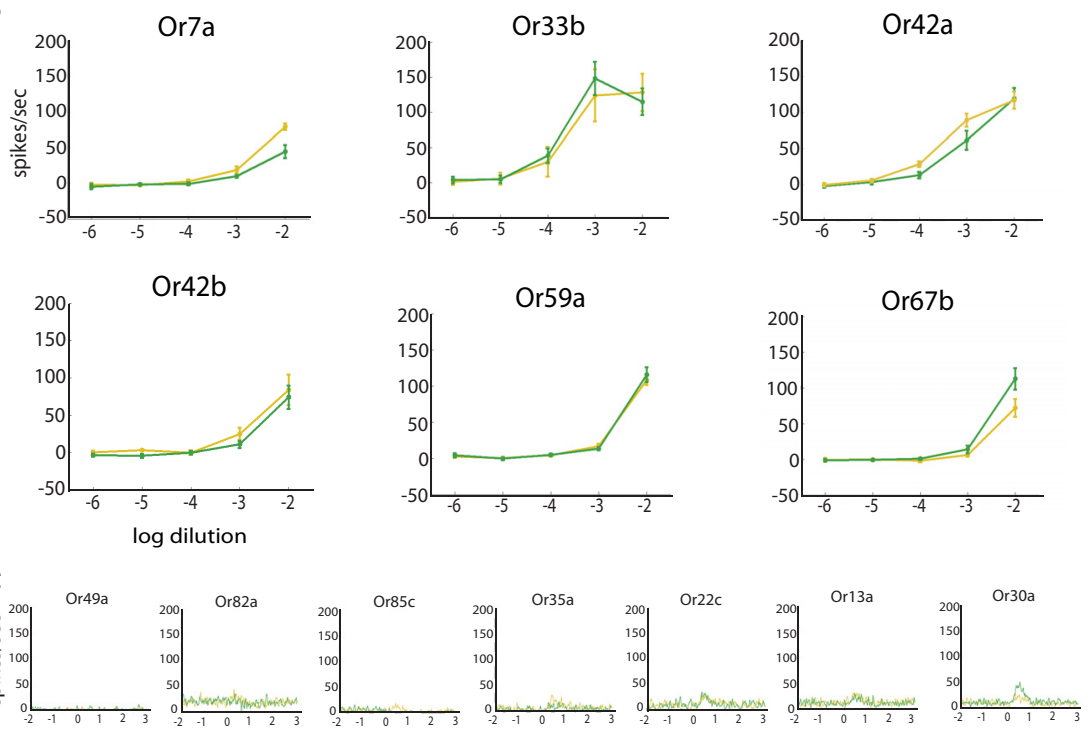
2-ethylpyrazine (yellow) and 2-methylpyrazine (green) at $10^{-2}$ dilutions. The two response profiles are indistinguishable (oneway ANOVA, $p=0.15$ ). $n=9$; error bars are SEM. We note that Or7a gave a greater mean response to 2-ethylpyrazine in this experiment, but not in the experiment shown in Table 1. Or67b gave a greater mean response to 2-methylpyrazine in this experiment, but not in the experiment shown in Table 1. $\boldsymbol{B}$, Dose-response curves for all receptors that showed a moderate response ( $\geq 50$ spikes/s) to at least one of the two odors at a $10^{-2}$ dilution. $n=9$, error bars are SEM. The data for the $10^{-2}$ dilution are the same as in $\boldsymbol{A}$. $\boldsymbol{C}$, Peristimulus time histograms of responses to 2-ethylpyrazine and 2-methylpyrazine at $10^{-2}$ dilutions. In each histogram, a solid line indicates the mean response and a shaded area represents the SEM, although the SEM is too small to be easily distinguished from the mean. $n=7-9$. The data are from the recordings used in $\boldsymbol{A}$.

\section{Supersustained responses to certain receptor-odorant combinations}

We were surprised to find that some responses to pyrazines were sustained for remarkably long periods. Typical excitatory responses returned to spontaneous firing levels within several seconds after the end of the odor stimulus period, as illustrated by the response elicited by 2,6-dimethylpyrazine from Or42a (Fig. $6 A$, top trace). By contrast, 2,6-dimethylpyrazine elicited a response from Or33b that lasted for minutes (Fig. 6A, second trace). Thus, the same odorant elicited a short-lasting response 


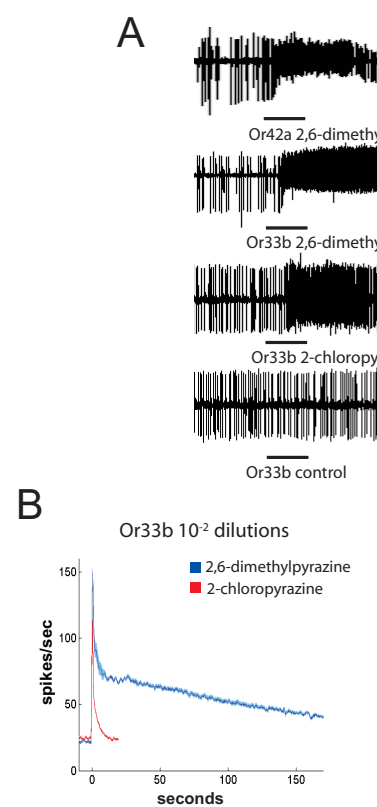

$E$
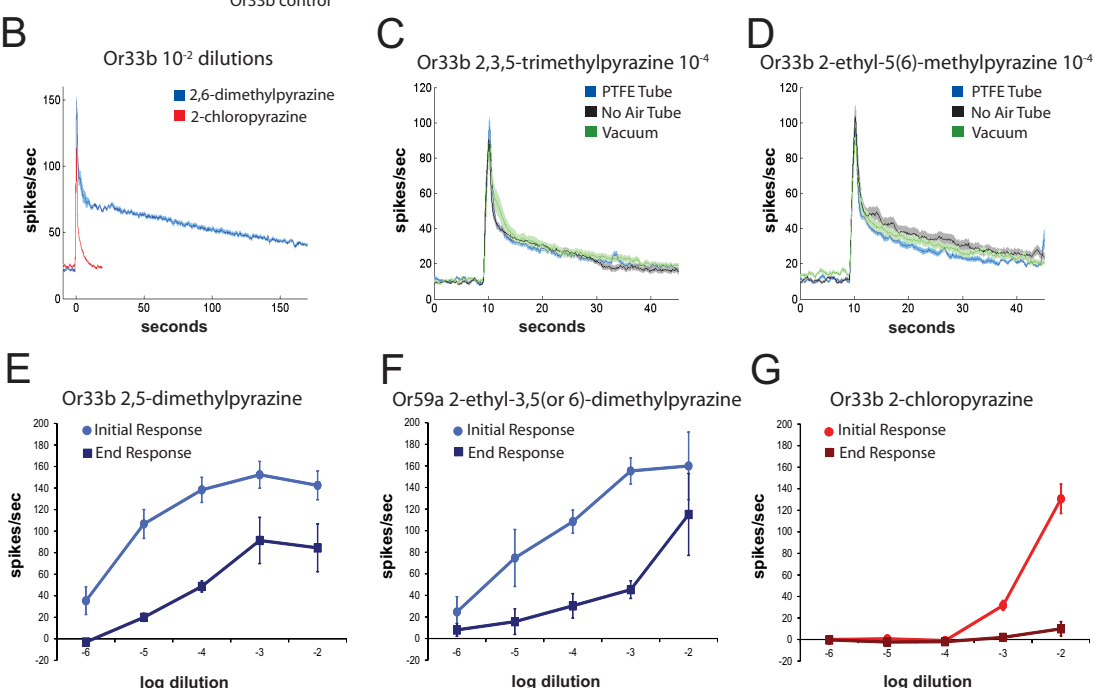

F

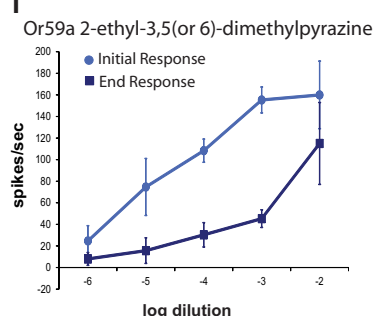

G

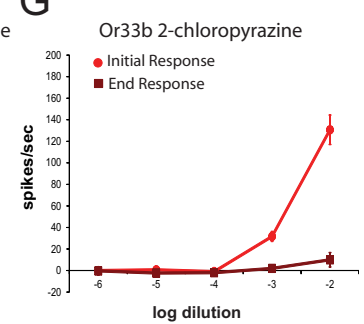

Figure 6. Supersustained responses. $\boldsymbol{A}$, Typical and supersustained responses to $10^{-2}$ odorant dilutions. The black bar represents the $0.5 \mathrm{~s}$ stimulus presentation period. In the control trace, the stimulus was the diluent alone. $\boldsymbol{B}$, Peristimulus time histograms of $0 \mathrm{r} 33 \mathrm{~b}$ excitatory responses. In each histogram, a solid line indicates the mean response and a shaded area represents the SEM, although the SEM is too small to be easily distinguished from the mean. $n=8-10$. C, D, Responses of $0 \mathrm{r} 33 \mathrm{~b}$ to $10^{-4}$ dilutions of two pyrazines following modification of the stimulus delivery system. $n=7-8$; shaded areas represent SEM. $\boldsymbol{E}-\boldsymbol{G}$, Dose-response curves of firing frequencies at dilutions ranging from $10^{-6}$ to $10^{-2}$. The initial frequency (circles) represents activity during the 0.5 sodor presentation period, and the final frequency (squares) represents activity during the $0.5 \mathrm{~s}$ period that begins $9 \mathrm{~s}$ after the beginning of the odor presentation period. The background firing rate and diluent response are subtracted from all responses. $E, n=4-6$; error bars are SEM. $F, n=4-6$; error bars are SEM. $\mathbf{G}, n=6$; error bars are SEM.

from one receptor and a long-lasting response from another, under the same conditions. We have termed the long-lasting responses "supersustained responses."

Supersustained responses are not only receptor specific, but also odorant specific. Whereas Or33b yielded a supersustained response to 2,6-dimethylpyrazine, it yielded a much shorter response to 2-chloropyrazine (Fig. 6A, second and third traces, and Fig. $6 B$ ). We note that these three odor-receptor combinations (illustrated in Fig. 6A, top three traces) all had comparable initial firing frequencies (Table 1 ).

We operationally defined a supersustained response as one in which the firing frequency was two SDs above the spontaneous firing frequency $9 \mathrm{~s}$ after the onset of the odor stimulus period. By this criterion, 9 of the Or33b responses and 10 of the Or59a responses were supersustained in our systematic analysis of the 525 pyrazine-receptor combinations (Fig. 3A, boxes). No other receptors yielded a supersustained response in our analysis. Supersustained responses appeared to decay initially with a very rapid decrease in frequency, followed by a gradual decay occurring over minutes (Fig. $6 \mathrm{~B}$, blue line).

We considered the possibility that supersustained responses arise as a consequence of long-lasting odor stimuli, as opposed to long-lasting responses per se; it seemed plausible that adherence of odorants to the glass tubing used in our delivery system and gradual release of the odorant from the glass could provoke a continuing response, especially if high doses of odorant were used as stimuli. To test this possibility, we tested different modes of stimulus presentation and lower concentrations of odorants.

We delivered a reduced odorant concentration, a $10^{-4}$ dilution of 2,3,5-trimethylpyrazine or 2-ethyl-5(6)-methylpyrazine, through tubing made of PTFE (of which Teflon is made) and found supersustained responses from Or33b for both (Fig. 6C,D). We then dispensed with tubing altogether, delivering a pulse of odor directly from the odor cartridge into the airstream directed at the antenna and then withdrawing the cartridge. The dynamics appeared to be unaffected by the absence of the odor delivery tube (Fig. $6 C, D$ ). Finally, we added a vacuum pump to promote withdrawal of odorant from the vicinity of the antenna, and again found no effect (Fig. $6 C, D)$. The simplest interpretation of these results is that a pulse of some odorants elicits a supersustained response from the system, in an odorant-specific and receptor-specific manner.

We next extended our investigation of whether supersustained responses could be elicited by lower concentrations of odorants. We measured initial action potential frequencies during the $0.5 \mathrm{~s}$ odor stimulus period, and we measured frequencies at $9 \mathrm{~s}$ after the onset of the stimulus period. We found that a supersustained response could be elicited from Or33b not only by a $10^{-2}$ dilution of 2,5-dimethylpyrazine, but also by $10^{-3}, 10^{-4}$, and $10^{-5}$ dilutions (Fig. $6 \mathrm{E}$, bottom line). At every concentration tested, the mean frequency of the end response was lower than during the initial period (Fig. $6 E$, top line), but even at a $10^{-5}$ dilution, the frequency exceeded that of the prestimulus spontaneous firing rate by more than two SDs. Likewise, 2-ethyl-3,5(or 6)-dimethylpyrazine elicited a supersustained response from Or59a at $10^{-2}, 10^{-3}$, and $10^{-4}$ dilutions (Fig. $6 \mathrm{~F}$, bottom line). As a control, we tested Or33b with 2-chloropyrazine, and found no supersustained responses at any dilution (Fig. 6G).

Finally, we considered the possibility that supersustained responses arise solely as a consequence of the ectopic expression of a receptor in a foreign environment. Tests of a variety of odorants with the native wild-type ab3A neuron (the neuron that is mutant in the empty neuron system, but that expresses the endogenous Or22a receptor in wild type) showed that methyl hexanoate at a $10^{-4}$ dilution yielded a supersustained response, arguing against this possibility (data not shown).

\section{Discussion}

We have systematically examined olfactory responses elicited by a closely related set of odorants. These odorants map to a 
region of odor space that has not previously been explored in such a manner.

Our analysis was motivated by a special interest in two fundamental issues. First, most previous analysis of odor coding has compared representations of highly diverse odorants. In nature, however, animals often encounter closely related odorants. Since similar odorants may have different biological significance for an animal, an understanding of differences in their coding is essential to an understanding of how they are discriminated. Second, odor space is vast, and it is not possible to examine all regions in detail. The present investigation is a case study of one region, and may serve as a model for others. The region we have studied is occupied by pyrazines, which are important to insects in general and are encountered by Drosophila in particular.

\section{Diversity of behavioral responses to pyrazines}

Pyrazines of the panel clustered in a physicochemical odor space. How does similarity in chemical properties relate to behavior? A priori, one can envision two extreme models. At one extreme, structurally similar odorants would elicit similar behavioral responses. At the other extreme, such a physicochemical odor space could exhibit a fractal property: the diversity of behavioral responses elicited by a set of odorants could be independent of the dimensions of the region of odor space that the odorants occupy.

We found that the behavioral responses to the pyrazines ranged widely, from exceptionally strong to essentially nil, along a smooth continuum. Thus, these chemically similar odors produced highly diverse responses. We note that in the present analysis, a single, simple behavioral parameter was examined. Further comparison of behavioral responses among pyrazines and dissimilar odorants should include an evaluation of other parameters such as navigational and postural descriptors.

\section{Coding of pyrazines by the larval Ors}

Different pyrazines elicited different patterns of response from the larval Or repertoire. Some pyrazines evoked moderate or strong activity from several Ors, with different pyrazines evoking activity from different combinations of Ors. Other pyrazines evoked little or no activity from any Ors.

Although the activity patterns elicited by different pyrazines are diverse, it is striking that most of the strong responses are from only two receptors. Or $33 \mathrm{~b}$ and Or59a accounted for 20 of the 27 strong responses (Fig. 3), with Or42a accounting for five of the others. Analysis of a diverse set of 26 odors in a previous study revealed strong responses from many more receptors (Kreher et al., 2008).

The strong response of Or33b to many pyrazines was of particular interest. Or $33 \mathrm{~b}$ did not respond to any of the structurally diverse odors tested in the previous study and thus may be tuned to pyrazines. A recent study of odor receptors from the malaria mosquito Anopheles gambiae showed that some of the most narrowly tuned receptors were robustly excited by odorants with high biological salience (Carey et al., 2010); it is possible that pyrazines may likewise be of particular biological importance to Drosophila. We note that Or59a also showed few moderate or strong responses to the broad odor panel used in the previous study (Kreher et al., 2008). Thus, it seems likely that behavioral responses to pyrazines are driven largely by receptors that do not provide major contributions to the responses to most other odorants.

\section{Two similar odors elicit similar physiology but different behavior}

One of the most striking results of this study concerns two odorants that are very similar in structure, 2-ethylpyrazine and 2-methylpyrazine. These odorants elicited extremely different behavioral responses, but remarkably similar responses from the Or repertoire. The Or responses were similar not only in terms of magnitude but also dynamics. Since behavioral output is ultimately driven by sensory input, the similarity in Or input elicited by the two odorants surprised us.

There are several possible explanations for the differences in behavioral response to the two odorants. First, it is possible that a small difference in the response magnitude of an Or to the two odorants is amplified so as to produce a large difference in the representation among higher-order neurons (Lei et al., 2004; Bhandawat et al., 2007; Olsen et al., 2007; Kazama and Wilson, 2008). The two odorants could also elicit subtle differences in the temporal patterns of spikes, which could in turn elicit major differences in behavior. Second, it is possible that some of the physiological responses we measure in our in vivo expression system do not reflect Or responses that occur in the behaving larva. For example, Or33b appears to be coexpressed with Or47a, and Or94a appears to be coexpressed with Or94b in the larva (Fishilevich et al., 2005), but not in our expression system [however, we note that the response profiles of some coexpressed receptors are additive (Ray et al., 2007), and neither Or $47 \mathrm{a}$ nor Or94b gave even moderate responses to any pyrazine in this study]. Third, it is possible that an allelic variant of an Or that we have not examined, but that is present in our larval population, confers different responses to 2-ethylpyrazine and 2-methylpyrazine. Finally, although we have not identified a correlation between vapor pressure and behavioral response, and although 2-ethylpyrazine elicits a greater response than 2-methylpyrazine over a broad concentration range despite its lower vapor pressure, we note that behavioral responses may be influenced by differences in spatial or temporal parameters of the odor gradients, which may in turn vary on account of different physicochemical properties of the odorants.

\section{Supersustained responses}

We have identified and characterized remarkably long-lasting physiological responses that are both odorant and receptor specific. Individual pyrazines elicited supersustained responses from some but not all of the receptors they activate; individual receptors gave supersustained responses to some but not all pyrazines that activate them. Supersustained responses are not restricted to very high doses of odorants; in at least one odorant-receptor combination, supersustained responses were observed from doses ranging over four orders of magnitude (Fig. $6 E$ ).

We were concerned that these long-lasting responses might reflect a long-lasting odor stimulus due to the nature of the stimulus delivery system and the physicochemical nature of the odorant; in particular, it seemed plausible that the responses might arise solely because of the adherence of odorants to the tubing of the delivery system. However, we found that some odorants gave supersustained responses even when administered without stationary tubing to which odorants could adhere. Nonetheless, we suspect that some supersustained responses observed in some experimental systems may in fact arise from the properties of the odorant and the design of the stimulus delivery system. Another consideration is that some odorants may adhere to biological surfaces, such as insect cuticle, and be released slowly. If so, this adherence is likely to occur in nature as well and may represent one aspect of how an odorant is encoded.

It is formally possible that some of the supersustained responses to pyrazines observed in our in vivo expression system do not occur in endogenous neurons. However, long-lasting responses have previously been reported in native moth ORNs (Kaissling et al., 1989), and responses that "greatly outlasted the 
duration of the odor stimulus" have been observed in locust ORNs (Raman et al., 2010). We observed supersustained responses in the native, wild-type ab3A neuron. Long-lasting responses have also been observed previously in ORNs that ectopically express either a fly (Bai and Carlson, 2010) or mosquito (Carey et al., 2010) receptor. In Caenorhabditis elegans, AWC olfactory neurons are stimulated by odor removal and show elevated $\mathrm{Ca}^{2+}$ levels for tens of seconds (Chalasani et al., 2007). Aplysia bag neurons respond to a relatively brief synaptic input with a response that lasts for up to $30 \mathrm{~min}$; this response is linked to egg-laying behavior (Hung and Magoski, 2007). Sustained action potential firing in response to a brief stimulus has also been documented in various regions of the mammalian brain (Major and Tank, 2004).

Supersustained responses in insect ORNs could in principle serve useful functions in odor coding. A long-lasting response could serve as a memory of a previously encountered odor. If a navigating insect loses track of an odor trail, such a memory could lead the animal to deploy a search strategy to relocate the odor.

It is also possible that some supersustained responses may impair navigation (Kramer, 1992) by preventing an ORN from reporting contemporaneous information about the local odor environment. In fact, it is possible that such sustained strong responses could impair the signaling of other ORNs via prolonged lateral inhibition among glomeruli in the antennal lobe (Olsen et al., 2010). If so, odorants that elicit supersustained responses could be useful in the control of insect pests and vectors of human disease by impairing their location of human hosts, food sources, and mates.

\section{References}

Abuin L, Bargeton B, Ulbrich MH, Isacoff EY, Kellenberger S, Benton R (2011) Functional architecture of olfactory ionotropic glutamate receptors. Neuron 69:44-60.

Al Abassi S, Birkett MA, Pettersson J, Pickett JA, Woodcock CM (1998) Ladybird beetle odour identified and found to be responsible for attraction between adults. Cell Mol Life Sci 54:876-879.

Ames JM, Elmore JS (1992) Aroma components of yeast extracts. Flavour Fragr J 7:89-103.

Bai L, Carlson JR (2010) Distinct functions of acj6 splice forms in odor receptor gene choice. J Neurosci 30:5028-5036.

Benton R, Vannice KS, Gomez-Diaz C, Vosshall LB (2009) Variant ionotropic glutamate receptors as chemosensory receptors in Drosophila. Cell 136:149-162.

Bhandawat V, Olsen SR, Gouwens NW, Schlief ML, Wilson RI (2007) Sensory processing in the Drosophila antennal lobe increases reliability and separability of ensemble odor representations. Nat Neurosci 10:14741482.

Carey AF, Wang G, Su CY, Zwiebel LJ, Carlson JR (2010) Odorant reception in the malaria mosquito Anopheles gambiae. Nature 464:66-71.

Chalasani SH, Chronis N, Tsunozaki M, Gray JM, Ramot D, Goodman MB, Bargmann CI (2007) Dissecting a circuit for olfactory behavior in Caenorhabditis elegans. Nature 450:63-70.

Couto A, Alenius M, Dickson BJ (2005) Molecular, anatomical, and functional organization of the Drosophila olfactory system. Curr Biol 15:1535-1547.

Dobritsa AA, van der Goes van Naters W, Warr CG, Steinbrecht RA, Carlson JR (2003) Integrating the molecular and cellular basis of odor coding in the Drosophila antenna. Neuron 37:827-841.

Fishilevich E, Domingos AI, Asahina K, Naef F, Vosshall LB, Louis M (2005) Chemotaxis behavior mediated by single larval olfactory neurons in Drosophila. Curr Biol 15:2086-2096.

Haddad R, Khan R, Takahashi YK, Mori K, Harel D, Sobel N (2008) A metric for odorant comparison. Nat Methods 5:425-429.

Hallem EA, Carlson JR (2006) Coding of odors by a receptor repertoire. Cell 125:143-160.

Hallem EA, Ho MG, Carlson JR (2004) The molecular basis of odor coding in the Drosophila antenna. Cell 117:965-979.
Hung AY, Magoski NS (2007) Activity-dependent initiation of a prolonged depolarization in Aplysia bag cell neurons: role for a cation channel. J Neurophysiol 97:2465-2479.

Kaissling KE, Meng LZ, Bestmann HJ (1989) Responses of bombykol receptor cells to $(Z, E)-4,6$-hexadecadiene and linalool. J Comp Physiol A 165:147-154.

Kazama H, Wilson RI (2008) Homeostatic matching and nonlinear amplification at identified central synapses. Neuron 58:401-413.

Kramer E (1992) Attractivity of pheromone surpassed by time-patterned application of two nonpheromone compounds. J Insect Behav 5:83-97.

Kreher SA, Kwon JY, Carlson JR (2005) The molecular basis of odor coding in the Drosophila larva. Neuron 46:445-456.

Kreher SA, Mathew D, Kim J, Carlson JR (2008) Translation of sensory input into behavioral output via an olfactory system. Neuron 59:110-124.

Lacey MJ, Allen MS, Harris RL, Brown WV (1991) Methoxypyrazines in sauvignon blanc grapes and wines. Am J Enol Vitic 42:103-108.

Larsson MC, Leal WS, Hansson BS (2001) Olfactory receptor neurons detecting plant odors and male volatiles in Anomala cuprea beetles (Coleoptera: Scarabaeidae). J Insect Physiol 47:1065-1076.

Larsson MC, Hallberg E, Kozlov MV, Francke W, Hansson BS, Löfstedt C (2002) Specialized olfactory receptor neurons mediating intra- and interspecific chemical communication in leafminer moths Erocrania spp. (Lepidoptera: Eriocraniidae). J Exp Biol 205:989-998.

Larsson MC, Domingos AI, Jones WD, Chiappe ME, Amrein H, Vosshall LB (2004) Or83b encodes a broadly expressed odorant receptor essential for Drosophila olfaction. Neuron 43:703-714.

Lei H, Christensen TA, Hildebrand JG (2004) Spatial and temporal organization of ensemble representations for different odor classes in the moth antennal lobe. J Neurosci 24:11108-11119.

Liu C, Pitts RJ, Bohbot JD, Jones PL, Wang G, Zwiebel LJ (2010) Distinct olfactory signaling mechanisms in the malaria vector mosquito Anopheles gambiae. PLoS Biol 8:e1000467.

Major G, Tank D (2004) Persistent neural activity: prevalence and mechanisms. Curr Opin Neurobiol 14:675-684.

Monte P, Woodard C, Ayer R, Lilly M, Sun H, Carlson J (1989) Characterization of the larval olfactory response in Drosophila and its genetic basis. Behav Genet 19:267-283.

Nikonov AA, Leal WS (2002) Peripheral coding of sex pheromone and a behavioral antagonist in the Japanese beetle, Popilla japonica. J Chem Ecol 28:1075-1089.

Nikonov AA, Peng G, Tsurupa G, Leal WS (2002) Unisex pheromone detectors and pheromone-binding proteins in scarab beetles. Chem Senses 27:495-504.

Olsen SR, Bhandawat V, Wilson RI (2007) Excitatory interaction between olfactory processing channels in the Drosophila antennal lobe. Neuron 54:89-103.

Olsen SR, Bhandawat V, Wilson RI (2010) Divisive normalization in olfactory population codes. Neuron 66:287-299.

Ramaekers A, Magnenat E, Marin EC, Gendre N, Jefferis GS, Luo L, Stocker RF (2005) Glomerular maps without cellular redundancy at successive levels of the Drosophila larval olfactory circuit. Curr Biol 15:982-992.

Raman B, Joseph J, Tang J, Stopfer M (2010) Temporally diverse firing patterns in olfactory receptor neurons underlie spatiotemporal neural codes for odors. J Neurosci 30:1994-2006.

Ray A, van der Goes van Naters W, Shiraiwa T, Carlson JR (2007) Mechanisms of odor receptor gene choice in Drosophila. Neuron 53:353-369.

Rodrigues V, Siddiqi O (1978) Genetic analysis of chemosensory pathway. Proc Indian Acad Sci B 87:147-160.

Sato K, Pellegrino M, Nakagawa T, Nakagawa T, Vosshall LB, Touhara K (2008) Insect olfactory receptors are heteromeric ligand-gated ion channels. Nature 452:1002-1006.

Vander Meer RK, Preston CA, Choi M (2010) Isolation of a pyrazine alarm pheromone component from the fire ant, Solenopsis invicta. J Chem Ecol $36: 163-170$

Wheeler JW, Blum MS (1973) Alkylpyrazine alarm pheromones in ponerine ants. Science 182:501-503.

Wicher D, Schäfer R, Bauernfeind R, Stensmyr MC, Heller R, Heinemann SH, Hansson BS (2008) Drosophila odorant receptors are both ligand-gated and cyclic-nucelotide-activated cation channels. Nature 452:1007-1011.

Woolfson A, Rothschild M (1990) Speculating about pyrazines. Proc Biol Sci 242:113-119. 